\title{
On the boundedness of the differentiation operator between weighted spaces of holomorphic functions
}

by

\author{
AnAhit HaRutyunyan (Yerevan) and Wolfgang Lusky (Paderborn)
}

\begin{abstract}
We give necessary and sufficient conditions on the weights $v$ and $w$ such that the differentiation operator $D: H v(\Omega) \rightarrow H w(\Omega)$ between two weighted spaces of holomorphic functions is bounded and onto. Here $\Omega=\mathbb{C}$ or $\Omega=\mathbb{D}$. In particular we characterize all weights $v$ such that $D: H v(\Omega) \rightarrow H w(\Omega)$ is bounded and onto where $w(r)=v(r)(1-r)$ if $\Omega=\mathbb{D}$ and $w=v$ if $\Omega=\mathbb{C}$. This leads to a new description of normal weights.
\end{abstract}

1. Introduction. Let $\Omega$ be the complex plane $\mathbb{C}$ or the open unit disk $\mathbb{D}=\{z \in \mathbb{C}:|z|<1\}$. A weight $v$ on $\Omega$ is a continuous non-increasing function $v:[0, a[\rightarrow] 0, \infty[$ where $a=1$ if $\Omega=\mathbb{D}$ and $a=\infty$ if $\Omega=\mathbb{C}$. We assume that $\lim _{r \rightarrow a} v(r)=0$ if $a=1$ and $\lim _{r \rightarrow a} r^{m} v(r)=0$ for all $m \geq 0$ if $a=\infty$. For a function $h: \Omega \rightarrow \mathbb{C}$ and $r \in[0, a[$ put

$$
M_{\infty}(h, r)=\sup _{|z|=r}|h(z)| \quad \text { and } \quad\|h\|_{v}=\sup _{0 \leq r<a} M_{\infty}(h, r) v(r) .
$$

We consider the Banach space

$$
H v(\Omega)=\left\{h: \Omega \rightarrow \mathbb{C} \text { holomorphic: }\|h\|_{v}<\infty\right\}
$$

endowed with the norm $\|\cdot\|_{v}$. Hence, a holomorphic function $h$ satisfies $h \in H v(\Omega)$ if and only if $M_{\infty}(h, r)=O(1 / v(r))$ as $r \rightarrow a$.

There is an extensive literature about the Banach spaces $H v(\Omega)$ and their generalisations to other domains $\Omega \subset \mathbb{C}^{n}$ or to corresponding spaces of harmonic functions (see e.g. [19, 20, 21, 2, 11, 8, 12, 15, 16, 14]). Moreover, many authors study special classes of operators between such spaces. For example, the authors of $[3,6,9]$ discuss multiplication operators $M_{\varphi} f=\varphi \cdot f$, $f \in H v(\Omega)$, where $\varphi$ is a fixed holomorphic function. Other papers $([7,5,22]$

2000 Mathematics Subject Classification: Primary 46E15; Secondary 47B38.

Key words and phrases: differentiation operator, holomorphic functions, weighted spaces.

Research of A. Harutyunyan supported by Deutsche Forschungsgemeinschaft 436 ARM 17/2/06. 
and many more) deal with composition operators $C_{\varphi} f=f \circ \varphi, f \in H v(\Omega)$, where $\varphi: \Omega \rightarrow \Omega$ is again a fixed holomorphic function.

Also, there is a vast literature on interpolation and sampling in these weighted spaces of holomorphic functions (e.g. [17, 18, 1, 10]). Here the operators

$$
T: H v(\mathbb{D}) \rightarrow l_{\infty}, \quad f \mapsto\left(f\left(z_{n}\right) v\left(z_{n}\right)\right)_{n},
$$

are studied where $\left(z_{n}\right)_{n} \subset \mathbb{D}$ is a given sequence, which is called a set of interpolation if $T$ is surjective and a sampling set if $T$ is a monomorphism. A nice survey of all these results is given in [4].

In our paper we discuss the question of what kind of growth condition the derivative $D h=h^{\prime}$ satisfies. In Section 2 we introduce necessary and sufficient conditions on weights $v$ and $w$ such that $D: H v(\Omega) \rightarrow H w(\Omega)$ is bounded and sometimes onto. In Section 3 we investigate the case $\Omega=\mathbb{D}$ and $w(r)=(1-r) v(r)$ while in Section 4 we focus on $\Omega=\mathbb{C}$ and $w=v$.

To this end we make some further assumptions on $v$ which do not restrict generality. We can always fix radii $r_{1}<r_{2}<\cdots<a$ such that $v\left(r_{n}\right)=$ $2 v\left(r_{n+1}\right)$ for all $n$ and change $v(r)$ keeping monotonicity for $r_{n}<r<$ $r_{n+1}$ without changing $H v(\Omega)$. Therefore we can always assume that $v$ is continuously differentiable. Moreover in the following, for any $n>0$, the function $\gamma_{n}(r)=r^{n} v(r)$ plays an important role. Put

$$
\begin{aligned}
& r_{n}=\min \left\{r: r \text { is a global maximum point of } \gamma_{n}\right\}, \\
& s_{n}=\max \left\{r: r \text { is a global maximum point of } \gamma_{n}\right\} .
\end{aligned}
$$

1.1. Lemma. If $m<n$ then $s_{m} \leq r_{n}$.

Proof. We have

$$
s_{m}^{n} v\left(s_{m}\right) \leq r_{n}^{n} v\left(r_{n}\right) \leq r_{n}^{n-m} s_{m}^{m} v\left(s_{m}\right) .
$$

Hence $s_{m}^{n-m} \leq r_{n}^{n-m}$ and thus $s_{m} \leq r_{n}$.

So, if $r_{m}<r<s_{m}$ then $r$ cannot be a global maximum point for $\gamma_{n}$ for any $n \neq m$. For those $m$ with $r_{m}<s_{m}$ we change $v$ on the interval $\left[r_{m}, s_{m}\right]$. Define $\widetilde{v}(r)=\left(r_{m} / r\right)^{m} v\left(r_{m}\right)$ if $r_{m}<r<s_{m}$. Then all $r \in\left[r_{m}, s_{m}\right]$ are global maximum points of $r^{m} \widetilde{v}(r)$ and we obtain $r^{m} \widetilde{v}(r)=r_{m}^{m} v\left(r_{m}\right)$. Moreover, $\widetilde{v}\left(r_{m}\right)=v\left(r_{m}\right)$ and $\widetilde{v}\left(s_{m}\right)=v\left(s_{m}\right)$. According to [16, Corollary $5.4],\|\cdot\|_{v}$ is equivalent to a norm which depends exclusively on the global maximum points of the functions $\gamma_{m}$. So in the following we assume that any $r \in\left[r_{m}, s_{m}\right]$, for any $m>0$, is a global maximum point of $\gamma_{m}$. This is no loss of generality, otherwise we go over to $\widetilde{v}$ where $\|\cdot\|_{\tilde{v}}$ is equivalent to $\|\cdot\|_{v}$.

1.2. Lemma. We have $\lim _{n \rightarrow \infty} r_{n}=a$.

Proof. According to Lemma 1.1, $r_{n}$ is increasing. Put $r=\lim _{n \rightarrow \infty} r_{n}$ and assume $r<a$. 
CASE $a=1$. Here we obtain

$$
1 \geq\left(\frac{2^{-1}(1+r)}{r_{n}}\right)^{n} \frac{v\left(2^{-1}(1+r)\right)}{v\left(r_{n}\right)} .
$$

Since

$$
\lim _{n \rightarrow \infty}\left(\frac{1}{2}\left(\frac{1}{r_{n}}+\frac{r}{r_{n}}\right)\right)^{n}=\infty \text { and } \lim _{n \rightarrow \infty} v\left(r_{n}\right)=v(r)
$$

we arrive at a contradiction.

CASE $a=\infty$. Here we have

$$
1 \geq\left(\frac{1+r}{r_{n}}\right)^{n} \frac{v(r+1)}{v\left(r_{n}\right)} \geq\left(\frac{1}{r}+1\right)^{n} \frac{v(r+1)}{v\left(r_{n}\right)} .
$$

Again we get a contradiction for large $n$.

According to our assumptions, any $r$ in $[0, a[$ is a global maximum point for some $\gamma_{n}$. We have $\gamma_{n}^{\prime}(r)=0$ if and only if $-r v^{\prime}(r) / v(r)=n$. Hence if $\gamma_{n}^{\prime}(r)=0$ then $\gamma_{m}^{\prime}(r) \neq 0$ for any $m \neq n$. This means that $r$ is a global maximum point of $\gamma_{n}$ and all local maximum points of $\gamma_{n}$ are also global.

2. The differentiation and integration operators $H v(\Omega) \rightarrow H w(\Omega)$ for general $w$. Let $v$ and $w$ be two weights. Assume that $H w(\Omega)$ is isomorphic to $l_{\infty}$. For each $n$ fix a maximum point $r_{n}$ of $r^{n} w(r)$. According to [16] there are numbers $0<m_{1}<m_{2}<\cdots, t_{n, k} \in \mathbb{R}$ and operators

$$
\left(T_{n} h\right)(z)=\sum_{m_{n-1} \leq k<m_{n+1}} t_{n, k} \alpha_{k} z^{k} \quad \text { for } h(z)=\sum_{k} \alpha_{k} z^{k}
$$

such that

$$
\begin{aligned}
c_{1} \sup _{n} \sup _{r_{m_{n-1}} \leq r \leq r_{m_{n+1}}} M_{\infty} & \left(T_{n} h, r\right) w(r) \leq\|h\|_{w} \\
& \leq c_{2} \sup _{n} \sup _{r_{m_{n-1}} \leq r \leq r_{m_{n+1}}} M_{\infty}\left(T_{n} h, r\right) w(r)
\end{aligned}
$$

for all $h \in H w(\Omega)$ and some $c_{1}, c_{2}>0$. Moreover there is a universal constant $\gamma>0$ such that

$$
M_{\infty}\left(T_{n} h, r\right) \leq \gamma M_{\infty}(h, r) \text { for all } n, h \text { and } r .
$$

Finally, either

$$
\sup _{n} \max \left(\left(\frac{r_{m_{n}}}{r_{m_{n-1}}}\right)^{m_{n}} \frac{w\left(r_{m_{n}}\right)}{w\left(r_{m_{n-1}}\right)},\left(\frac{r_{m_{n-1}}}{r_{m_{n}}}\right)^{m_{n-1}} \frac{w\left(r_{m_{n-1}}\right)}{w\left(r_{m_{n}}\right)}\right)<\infty
$$

and

$$
0<\inf _{n} \frac{m_{n+1}-m_{n}}{m_{n}-m_{n-1}} \leq \sup _{n} \frac{m_{n+1}-m_{n}}{m_{n}-m_{n-1}}<\infty
$$

or

$$
\sup \left(m_{n+1}-m_{n-1}\right)<\infty \text {. }
$$


In the latter case we can split $T_{n}$ further, i.e. we can assume

$$
d_{1} \sup _{n \in \mathbb{Z}_{+}}\left|\alpha_{n}\right| r_{n}^{n} w\left(r_{n}\right) \leq\|h\|_{w} \leq d_{2} \sup _{n \in \mathbb{Z}_{+}}\left|\alpha_{n}\right| r_{n}^{n} w\left(r_{n}\right)
$$

for some $d_{1}, d_{2}>0$ and all $h=\sum_{k} \alpha_{k} z^{k} \in H w(\Omega)$. ((4) is not possible for $\Omega=\mathbb{D}$, see [15].)

If (3) holds then we have

$$
\sup _{n}\left(\frac{r_{m_{n}}}{r_{m_{n-1}}}\right)^{m_{n}-m_{n-1}}<\infty .
$$

Now [16, Lemma 3.1] implies, for any $r \in\left[r_{m_{n-1}}, r_{m_{n+1}}\right]$,

$$
\begin{aligned}
& M_{\infty}\left(T_{n} h, r\right) w(r) \leq 2\left(\frac{r}{r_{m_{n+1}}}\right)^{m_{n-1}} \frac{w(r)}{w\left(r_{m_{n+1}}\right)} M_{\infty}\left(T_{n} h, r_{m_{n+1}}\right) w\left(r_{m_{n+1}}\right) \\
& \leq 2\left(\frac{r_{m_{n-1}}}{r_{m_{n+1}}}\right)^{m_{n-1}} \frac{w\left(r_{m_{n-1}}\right)}{w\left(r_{m_{n+1}}\right)} M_{\infty}\left(T_{n} h, r_{m_{n+1}}\right) w\left(r_{m_{n+1}}\right) \\
&=2\left(\frac{r_{m_{n-1}}}{r_{m_{n}}}\right)^{m_{n-1}} \frac{w\left(r_{m_{n-1}}\right)}{w\left(r_{m_{n}}\right)}\left(\frac{r_{m_{n}}}{r_{m_{n+1}}}\right)^{m_{n}} \frac{w\left(r_{m_{n}}\right)}{w\left(r_{m_{n+1}}\right)}\left(\frac{r_{m_{n+1}}}{r_{m_{n}}}\right)^{m_{n}-m_{n-1}} \\
& \quad \times M_{\infty}\left(T_{n} h, r_{m_{n+1}}\right) w\left(r_{m_{n+1}}\right) \\
& \leq d M_{\infty}\left(T_{n} h, r_{m_{n+1}}\right) w\left(r_{m_{n+1}}\right)
\end{aligned}
$$

for some universal constant $d>0$.

For the last inequality we used (3), $\left(3^{\prime}\right)$ and (6). (According to $\left(3^{\prime}\right)$ there is a universal constant $c$ with $m_{n}-m_{n-1} \leq c\left(m_{n+1}-m_{n}\right)$ for all $n$. Hence

$$
\left(\frac{r_{m_{n+1}}}{r_{m_{n}}}\right)^{m_{n}-m_{n-1}} \leq\left(\left(\frac{r_{m_{n+1}}}{r_{m_{n}}}\right)^{m_{n+1}-m_{n}}\right)^{c},
$$

and this is uniformly bounded by (6).)

Therefore, in this case, (1) implies

$$
\begin{aligned}
\widetilde{c}_{1} \sup _{n} M_{\infty}\left(T_{n} h, r_{m_{n+1}}\right) w\left(r_{m_{n+1}}\right) & \leq\|h\|_{w} \\
& \leq \widetilde{c}_{2} \sup _{n} M_{\infty}\left(T_{n} h, r_{m_{n+1}}\right) w\left(r_{m_{n+1}}\right)
\end{aligned}
$$

for some $\widetilde{c}_{1}, \widetilde{c}_{2}>0$.

It is known that $H w(\Omega)$ is isomorphic to $l_{\infty}$ if and only if

$$
\begin{aligned}
& \forall b_{1}>1 \exists b_{2}>1 \exists c>0 \forall m, n \geq c, \\
& \qquad \begin{aligned}
|m-n| \geq c \text { and }\left(\frac{r_{m}}{r_{n}}\right)^{m} \frac{w\left(r_{m}\right)}{w\left(r_{n}\right)} \leq b_{1} \\
\Rightarrow\left(\frac{r_{n}}{r_{m}}\right)^{n} \frac{w\left(r_{n}\right)}{w\left(r_{m}\right)} \leq b_{2} .
\end{aligned}
\end{aligned}
$$


Examples include $(1-r)^{\alpha}, \alpha>0, \exp \left(-(1-r)^{-1}\right)$ on $\mathbb{D}, \exp (-\alpha r)$, $\exp \left(-\log ^{2} r\right)$ on $\mathbb{C}$.

If $H w(\Omega)$ is not isomorphic to $l_{\infty}$ then it is isomorphic to the space $H_{\infty}=\{h: \mathbb{D} \rightarrow \mathbb{C}: h$ holomorphic and bounded $\}$ (see [16]). Here we still obtain estimates similar to (1) but (2)-(4) will fail to hold.

Now we investigate the differentiation operator $D: H v(\Omega) \rightarrow H w(\Omega)$. Let $h(z)=\sum_{k} \alpha_{k} z^{k}$. For $n>0$ define the Cesàro mean $\sigma_{n}$ by

$$
\left(\sigma_{n} h\right)(z)=\sum_{k \leq n} \frac{[n]-k}{[n]} \alpha_{k} z^{k},
$$

where $[n]$ is the largest integer $\leq n$. Moreover, for $j \in \mathbb{Z}$, define the shift $U_{j}$ by

$$
\left(U_{j} h\right)\left(r e^{i \varphi}\right)=\sum_{k} \alpha_{k} r^{k} e^{i(k+j) \varphi} .
$$

We formally extend the definition of $T_{n}$ to $T_{n} U_{j} h$ by putting

$$
\left(T_{n} U_{j} h\right)\left(r e^{i \varphi}\right)=\sum_{m_{n-1} \leq k+j<m_{n}} t_{n, k} \alpha_{k} r^{k} e^{i(k+j) \varphi} .
$$

Define $g\left(\varrho e^{i \varphi}\right)=\left(U_{j} h\right)\left(\varrho r e^{i \varphi}\right)$. Then (2) applied to $g$ with $\varrho=1$ implies $M_{\infty}\left(T_{n} U_{j} h, r\right) \leq \gamma M_{\infty}\left(U_{j} h, r\right) \leq \gamma M_{\infty}(h, r) \quad$ for all $r$ and $n$.

2.1. THEOREM.

(a) Let $H w(\Omega)$ be isomorphic to $l_{\infty}$. If

$$
\limsup _{r \rightarrow a}\left(-\frac{w^{\prime}(r)}{v(r)}\right)<\infty
$$

then $D: H v(\Omega) \rightarrow H w(\Omega)$ is bounded.

(b) Let $s_{n}$ be a global maximum point of $r^{n} v(r)$. If $D: H v(\Omega) \rightarrow H w(\Omega)$ is bounded then

$$
\limsup _{n \in \mathbb{Z}_{+}, n \rightarrow \infty}\left(-\frac{v^{\prime}\left(s_{n}\right)}{v^{2}\left(s_{n}\right)} w\left(s_{n}\right)\right)<\infty .
$$

If , in addition, $\lim \sup _{n \in \mathbb{Z}_{+}, n \rightarrow \infty} s_{n+1} / s_{n}<\infty$, then also

$$
\limsup _{r \rightarrow a}\left(-\frac{v^{\prime}(r)}{v^{2}(r)} w(r)\right)<\infty .
$$

(In (b), Hw( $\Omega$ ) need not be isomorphic to $l_{\infty}$.)

Proof. (a) Fix $n$. Assume that (7) holds. Then it suffices to consider $M_{\infty}\left(T_{n} D h, r_{m_{n+1}}\right) w\left(r_{m_{n+1}}\right)$. We have

$$
\left(T_{n} D h\right)(z)=\frac{m_{n+1}}{r_{m_{n+1}}}\left(U_{-1}\left(\mathrm{id}-\sigma_{m_{n+1}}\right) U_{1} T_{n} U_{-1} h\right)(z)
$$


if $|z|=r_{m_{n+1}}$. The operators $U_{k}, k= \pm 1, T_{n} U_{-1}$ and $\sigma_{m_{n+1}}$ are uniformly bounded with respect to $M_{\infty}(\cdot, r)$ for all $r$ and the operator norms do not depend on $r$. Hence there is a universal constant $c$ with

$$
M_{\infty}\left(T_{n} D h, r_{m_{n+1}}\right) w\left(r_{m_{n+1}}\right) \leq c \frac{m_{n+1}}{r_{m_{n+1}}} M_{\infty}\left(h, r_{m_{n+1}}\right) w\left(r_{m_{n+1}}\right) .
$$

On the other hand we have $\left.\left(r^{m_{n+1}} w(r)\right)^{\prime}\right|_{r=r_{m_{n+1}}}=0$ since $r_{m_{n+1}}$ is a global maximum point of $r^{m_{n+1}} w(r)$. This implies $m_{n+1} w\left(r_{m_{n+1}}\right) / r_{m_{n+1}}=$ $-w^{\prime}\left(r_{m_{n+1}}\right)$. Fix some $r_{0}>0$. A change of $v$ and $w$ on $\left[0, r_{0}\right]$ does not affect $H v(\Omega)$ and $H w(\Omega)$. Therefore we can assume that there is $d>0$ with $-w^{\prime}(r) / v(r) \leq d$ for all $r$. Then we obtain

$$
M_{\infty}\left(T_{n} D h, r_{m_{n+1}}\right) w\left(r_{m_{n+1}}\right) \leq c d M_{\infty}\left(h, r_{m_{n+1}}\right) v\left(r_{m_{n+1}}\right) \leq c d\|h\|_{v} .
$$

By (7), $D$ is bounded. The proof for the case (5) is the same.

(b) Fix $r>0$. According to our general assumption $r$ is a global maximum point for some function $r^{n} v(r)$. Hence we have $s_{[n]} \leq r \leq s_{[n]+1}$ with $n=-r v^{\prime}(r) / v(r)$. Assume that $r$ is so large that $1 \leq[n]$. Consider $h(z)=z^{[n]}$. We have

$$
\|h\|_{v}=s_{[n]}^{[n]} v\left(s_{[n]}\right) \leq\left(\frac{r}{s_{[n]}}\right)^{n-[n]} r^{[n]} v(r)
$$

and

$$
[n] r^{[n]-1} w(r) \leq\|D h\|_{w} \leq\|D\| \cdot\|h\|_{v} \leq\|D\|\left(\frac{r}{s_{[n]}}\right)^{n-[n]}{ }^{[n]} v(r) .
$$

Hence

$$
\frac{n}{r} \frac{w(r)}{v(r)} \leq \frac{n}{[n]}\|D\|\left(\frac{r}{s_{[n]}}\right)^{n-[n]}
$$

and therefore

$$
-\frac{v^{\prime}(r)}{v^{2}(r)} w(r) \leq \frac{n}{[n]}\|D\|\left(\frac{r}{s_{[n]}}\right)^{n-[n]} \leq \frac{n}{[n]}\|D\|\left(\frac{s_{[n]+1}}{s_{[n]}}\right)^{n-[n]} .
$$

For $r=s_{[n]}$ we obtain the first assertion of (b). If $\sup _{n} s_{[n]+1} / s_{[n]}<\infty$ then the second assertion of (b) follows.

Recall that $\lim \sup _{n \in \mathbb{Z}_{+}, n \rightarrow \infty} s_{n+1} / s_{n}<\infty$ always holds if $\Omega=\mathbb{D}$.

Let $I$ be the integration operator, i.e. for a holomorphic function $h$ we put

$$
(I h)(z)=\int_{0}^{z} h(u) d u
$$

To decide whether the differentiation operator is surjective we prove 


\subsection{Proposition.}

(a) Let $H w(\Omega)$ be isomorphic to $l_{\infty}$. Moreover, assume that

$$
\limsup _{r \rightarrow a}\left(-\frac{w^{2}(r)}{w^{\prime}(r) v(r)}\right)<\infty .
$$

Then $I: H v(\Omega) \rightarrow H w(\Omega)$ is bounded.

(b) Let $s_{n}$ be a global maximum point of $r^{n} v(r)$. If $I: H v(\Omega) \rightarrow H w(\Omega)$ is bounded then

$$
\limsup _{n \in \mathbb{Z}_{+}, n \rightarrow \infty}\left(-\frac{w\left(s_{n}\right)}{v^{\prime}\left(s_{n}\right)}\right)<\infty .
$$

If , moreover, $\lim \sup _{n \in \mathbb{Z}_{+}, n \rightarrow \infty} s_{n+1} / s_{n}<\infty$ then also

$$
\limsup _{r \rightarrow a}\left(-\frac{w(r)}{v^{\prime}(r)}\right)<\infty .
$$

(In (b), $H w(\Omega)$ need not be isomorphic to $l_{\infty}$.)

Proof. (a) We use (7) again. (The proof for the case (5) is the same.) Fix $n$ and consider $h \in H v(\Omega)$. We have

$$
\left(T_{n} I h\right)\left(r e^{i \varphi}\right)=\int_{0}^{r}\left(T_{n} U_{1} h\right)\left(s e^{i \varphi}\right) d s .
$$

Using [16, Lemma 3.1(a)], we see that, for any $s \in\left[r_{m_{n-1}}, r_{m_{n+1}}\right]$,

$$
M_{\infty}\left(T_{n} U_{1} h, s\right)=M_{\infty}\left(U_{-1} T_{n} U_{1} h, s\right) \leq\left(\frac{s}{r_{m_{n-1}}}\right)^{m_{n+1}} M_{\infty}\left(T_{n} U_{1} h, r_{m_{n-1}}\right) .
$$

In particular,

$$
\begin{aligned}
& M_{\infty}\left(T_{n} U_{1} h, r_{m_{n+1}}\right) w\left(r_{m_{n+1}}\right) \\
& \leq\left(\frac{r_{m_{n+1}}}{r_{m_{n-1}}}\right)^{m_{n+1}} \frac{w\left(r_{m_{n+1}}\right)}{w\left(r_{m_{n-1}}\right)} M_{\infty}\left(T_{n} U_{1} h, r_{m_{n-1}}\right) w\left(r_{m_{n-1}}\right) \\
&=\left(\frac{r_{m_{n+1}}}{r_{m_{n}}}\right)^{m_{n+1}} \frac{w\left(r_{m_{n+1}}\right)}{w\left(r_{m_{n}}\right)}\left(\frac{r_{m_{n}}}{r_{m_{n-1}}}\right)^{m_{n}} \frac{w\left(r_{m_{n}}\right)}{w\left(r_{m_{n-1}}\right)} \\
& \cdot\left(\frac{r_{m_{n}}}{r_{m_{n-1}}}\right)^{m_{n+1}-m_{n}} M_{\infty}\left(T_{n} U_{1} h, r_{m_{n-1}}\right) w\left(r_{m_{n-1}}\right) \\
& \leq d M_{\infty}\left(T_{n} U_{1} h, r_{m_{n-1}}\right) w\left(r_{m_{n-1}}\right)
\end{aligned}
$$

for some universal constant $d$. (As before, the last inequality follows from $(3),\left(3^{\prime}\right)$ and (6).) Hence in view of (7) it suffices to consider the right-hand side of the preceding inequality. We have, using [16, Lemma 3.1(b)],

$$
M_{\infty}\left(T_{n} U_{1} h, s\right) \leq 2\left(\frac{s}{r_{m_{n-1}}}\right)^{m_{n-1}-1} M_{\infty}\left(T_{n} U_{1} h, r_{m_{n-1}}\right)
$$


if $s \leq r_{m_{n-1}}$. (Recall that $M_{\infty}\left(T_{n} U_{1} h, r\right)=M_{\infty}\left(U_{-1} T_{n} U_{1} h, r\right)$ for any $r$, and $\bar{U}_{-1} T_{n} U_{1} h$ has the form $\left(U_{-1} T_{n} U_{1} h\right)\left(r e^{i \varphi}\right)=\sum_{k \geq m_{n-1}-1} \alpha_{k} r^{k} e^{i k \varphi}$ for some $\alpha_{k}$.) This implies

$$
\begin{gathered}
M_{\infty}\left(T_{n} I h, r_{m_{n-1}}\right) w\left(r_{m_{n-1}}\right) \leq \int_{0}^{r_{m_{n-1}}} M_{\infty}\left(T_{n} U_{1} h, s\right) d s w\left(r_{m_{n-1}}\right) \\
\leq 2 M_{\infty}\left(T_{n} U_{1} h, r_{m_{n-1}}\right) \int_{0}^{r_{m_{n-1}}}\left(\frac{s}{r_{m_{n-1}}}\right)^{m_{n-1}-1} d s w\left(r_{m_{n-1}}\right) \\
\leq c M_{\infty}\left(h, r_{m_{n-1}}\right) \frac{r_{m_{n-1}}}{m_{n-1}} w\left(r_{m_{n-1}}\right)
\end{gathered}
$$

where $c$ is a universal constant. Since $r_{m_{n-1}} / m_{n-1}=-w\left(r_{m_{n-1}}\right) / w^{\prime}\left(r_{m_{n-1}}\right)$ we conclude that

$$
M_{\infty}\left(T_{n} I h, r_{m_{n-1}}\right) w\left(r_{m_{n-1}}\right) \leq c M_{\infty}\left(h, r_{m_{n-1}}\right)\left(-\frac{w^{2}\left(r_{m_{n-1}}\right)}{w^{\prime}\left(r_{m_{n-1}}\right)}\right) .
$$

Our assumptions yield a universal constant $c_{1}$ and $r_{0}>0$ with $-w^{2}(r) / w^{\prime}(r)$ $\leq c_{1} v(r)$ for all $r \geq r_{0}$. We may assume again $r_{0}=0$ (and perhaps change $v$ and $w$ on $\left.\left[0, r_{0}\right]\right)$. Then $\|I h\|_{w} \leq d_{1}\|h\|_{v}$ for some universal constant $d_{1}$.

(b) Fix $r>0$. Then there is $n>0$ such that $r$ is a global maximum point of $r^{n} v(r)$. We have $s_{[n]} \leq r \leq s_{[n]+1}$. With $h(z)=z^{[n]}$ we obtain $\|h\|_{v}=s_{[n]}^{[n]} v\left(s_{[n]}\right)$ and

$$
\frac{1}{[n]+1} r^{[n]+1} w(r) \leq\|I h\|_{w} \leq\|I\| s_{[n]}^{[n]} v\left(s_{[n]}\right) \leq\|I\|\left(\frac{r}{s_{[n]}}\right)^{n-[n]} r^{[n]} v(r) .
$$

This yields

$$
\frac{r}{n} \frac{w(r)}{v(r)} \leq \frac{[n]+1}{n}\left(\frac{r}{s_{[n]}}\right)^{n-[n]}\|I\| \leq \frac{[n]+1}{n}\left(\frac{s_{[n]+1}}{s_{[n]}}\right)^{n-[n]}\|I\|
$$

and hence

$$
-\frac{w(r)}{v^{\prime}(r)} \leq \frac{[n]+1}{n}\left(\frac{r}{s_{[n]}}\right)^{n-[n]}\|I\| \leq \frac{[n]+1}{n}\left(\frac{s_{[n]+1}}{s_{[n]}}\right)^{n-[n]}\|I\|,
$$

which implies (b).

2.3. Corollary. Assume that $H v(\Omega)$ and $H w(\Omega)$ are isomorphic to $l_{\infty}$. If

$$
\limsup _{r \rightarrow a}\left(-\frac{w^{\prime}(r)}{v(r)}\right)<\infty \quad \text { and } \quad \limsup _{r \rightarrow a}\left(-\frac{v^{2}(r)}{v^{\prime}(r) w(r)}\right)<\infty
$$

then $D: H v(\Omega) \rightarrow H w(\Omega)$ is bounded and surjective. 
Proof. The boundedness follows from Theorem 2.1. According to Proposition 2.2. the integration operator $I: H w(\Omega) \rightarrow H v(\Omega)$ is bounded, which yields the surjectivity of $D$.

We deduce that, in view of the open mapping theorem, under the assumptions of Corollary 2.3 there are universal constants $c$ and $d$ such that $c\|h\|_{v} \leq\left\|h^{\prime}\right\|_{w} \leq d\|h\|_{v}$ whenever $h \in H v(\Omega)$ and $h(0)=0$.

\section{The differentiation operator on holomorphic functions over} the unit disk. Here we consider $\Omega=\mathbb{D}$. First we show that $D$ is never a bounded endomorphism $H v(\mathbb{D}) \rightarrow H v(\mathbb{D})$.

3.1. Proposition. For any weight $v$ there exists a function $h \in H v(\mathbb{D})$ such that $h^{\prime} \notin H v(\mathbb{D})$.

Proof. Otherwise we would have $D(H v(\mathbb{D})) \subset H v(\mathbb{D})$ and, in view of the closed graph theorem, $D: H v(\mathbb{D}) \rightarrow H v(\mathbb{D})$ would be bounded. If $r_{n}$ is a global maximum point of $r^{n} v(r)$ we would obtain $n r_{n}^{n-1} v\left(r_{n}\right) \leq\|D\| r_{n}^{n} v\left(r_{n}\right)$ for any $n \in \mathbb{Z}_{+}$. Hence $n /\|D\| \leq r_{n} \leq 1$ for all $n$, a contradiction.

If we consider $w(r)=(1-r) v(r)$ we obtain positive results. We extend Theorem 3.1 of [13].

3.2. Theorem. Let $v:[0,1[\rightarrow] 0, \infty[$ be a weight and put $w(r)=$ $(1-r) v(r)$. Then the following are equivalent:

(i) If $h \in H v(\mathbb{D})$ then $h^{\prime} \in H w(\mathbb{D})$.

(ii) $D: H v(\mathbb{D}) \rightarrow H w(\mathbb{D})$ is bounded.

(iii) $\limsup _{r \rightarrow 1}\left(-\frac{(1-r) v^{\prime}(r)}{v(r)}\right)<\infty$.

(iv) $v(r) /(1-r)^{\alpha}$ is increasing on $\left[r_{0}, 1\left[\right.\right.$ for some $\alpha>0$ and $r_{0}>0$.

(v) $\sup _{n} \frac{v\left(1-2^{-n}\right)}{v\left(1-2^{-n-1}\right)}<\infty$.

Proof. (i) $\Rightarrow$ (ii) follows from the closed graph theorem; (ii) $\Rightarrow$ (i) is obvious; (ii) $\Rightarrow$ (iii) is a consequence of Theorem 2.1(b).

(iii) $\Leftrightarrow\left(\right.$ iv): Consider $f(r)=\log \left(v(r)(1-r)^{-\alpha}\right)$. Then

$$
f^{\prime}(r)=\left(\alpha+(1-r) \frac{v^{\prime}(r)}{v(r)}\right) \frac{1}{1-r},
$$

which proves the claim.

(iv) $\Rightarrow(v)$ follows from $[10$, Lemma $1(\mathrm{a})]$, and $(\mathrm{v}) \Rightarrow(\mathrm{i})$ from $[13$, Theorem 3.1].

Property (iv) of the preceding theorem is known as property (U) (see [10]).

To round out the discussion we mention the following result which was essentially proved in [13]. 
3.3. Theorem. Let $v:[0,1[\rightarrow] 0, \infty[$ be a weight and put $w(r)=$ $(1-r) v(r)$. Then the following are equivalent:

(i) $h \in H v(\mathbb{D})$ if and only if $h^{\prime} \in H w(\mathbb{D})$.

(ii) $0<\liminf _{r \rightarrow 1}\left(-\frac{(1-r) v^{\prime}(r)}{v(r)}\right)<\limsup _{r \rightarrow 1}\left(-\frac{(1-r) v^{\prime}(r)}{v(r)}\right)<\infty$.

(iii) $v(r) /(1-r)^{\alpha}$ is increasing and $v(r) /(1-r)^{\beta}$ is decreasing on $\left[r_{0}, 1[\right.$ for some $\alpha>0, \beta>0$ and $r_{0}>0$.

(iv) $\sup _{n} \frac{v\left(1-2^{-n}\right)}{v\left(1-2^{-n-1}\right)}<\infty$ and $\limsup _{n} \frac{v\left(1-2^{-n-k}\right)}{v\left(1-2^{-n}\right)}<1$ for some $k \in \mathbb{Z}_{+}$.

Proof. (ii) $\Leftrightarrow$ (iii): Put

$$
f(r)=\log \left(\frac{v(r)}{(1-r)^{\alpha}}\right) \quad \text { and } \quad g(r)=\log \left(\frac{v(r)}{(1-r)^{\beta}}\right) .
$$

Then

$$
f^{\prime}(r)=\left(\alpha+(1-r) \frac{v^{\prime}(r)}{v(r)}\right) \frac{1}{1-r} \quad \text { and } \quad g^{\prime}(r)=\left(\beta+(1-r) \frac{v^{\prime}(r)}{v(r)}\right) \frac{1}{1-r} .
$$

From this we derive the claim.

(iii) $\Leftrightarrow$ (iv) is [10, Lemma 1$]$.

(iv) $\Rightarrow$ (i) is [13, Theorem 3.1].

(i) $\Rightarrow$ (iv): According to Theorem 3.2 we have

$$
\sup _{n} \frac{v\left(1-2^{-n}\right)}{v\left(1-2^{-n-1}\right)}<\infty .
$$

Then [13, Theorem 3.1] yields (iv).

Weights $v$ with property (iii) of the preceding theorem are called normal (see [19]). Note that here $\|h\|_{v}$ is equivalent to $|h(0)|+\sup _{0 \leq r<1} M_{\infty}\left(h^{\prime}, r\right)$ $\times(1-r) v(r)$.

EXAMPles. $v(r)=(1-r)^{\alpha}$ for some $\alpha>0$ satisfies the assumptions of Theorem 3.3; $v(r)=(1-\log (1-r))^{-1}$ satisfies the assumptions of Theorem 3.2 but not of Theorem $3.3 ; v(r)=\exp \left(-(1-r)^{-1}\right)$ does not even satisfy the assumptions of Theorem 3.2.

4. The differentiation operator on entire functions. In contrast to $\mathbb{D}$, for $\Omega=\mathbb{C}$, we may have $D H v(\Omega) \subset H v(\Omega)$. We characterize these weights. To this end we recall some facts for general weights $v$. Proposition 5.2 and Lemma 5.3 of [16] imply that there are constants $c_{1}, c_{2}>0$, integers $0 \leq k_{1}<k_{2}<\cdots$, radii $0<t_{1}<t_{2}<\cdots$ and numbers $s_{n, j}>0$ 
such that the operators $T_{n}$ with

$$
\left(T_{n} h\right)(z)=\sum_{k_{n-2}<j \leq k_{n+1}} s_{n, j} \alpha_{j} z^{j} \quad \text { for } h(z)=\sum_{j} \alpha_{j} z^{j}
$$

satisfy

$$
c_{1} \sup _{n} M_{\infty}\left(T_{n} h, t_{n}\right) v\left(t_{n}\right) \leq\|h\|_{v} \leq c_{2} \sup _{n} M_{\infty}\left(T_{n} h, t_{n}\right) v\left(t_{n}\right)
$$

for all $h \in H v(\Omega)$. Moreover

$$
\|h\|_{v} \leq c_{2} M_{\infty}\left(h, t_{n}\right) v\left(t_{n}\right) \quad \text { whenever } \quad h \in T_{n} H v(\Omega)
$$

and $n=1,2, \ldots$ (see also [16, Corollary 4.4]). Finally, the numbers $s_{n, j}$ are such that the shifts $U_{ \pm 1}$ satisfy

$$
M_{\infty}\left(\left(T_{n}-U_{-k} T_{n} U_{k}\right) h, r\right) \leq \gamma M_{\infty}(h, r) \quad \text { for all } n, h, r \text { and } k= \pm 1
$$

where $\gamma>0$ is a universal constant. (See the operators of [16, Lemma 5.3].)

4.1. TheOREM. Let $v:[0, \infty[\rightarrow] 0, \infty[$ be a weight. Then the following are equivalent:

(i) $D: H v(\mathbb{C}) \rightarrow H v(\mathbb{C})$ is bounded,

(ii) $\limsup _{r \rightarrow \infty}\left(-v^{\prime}(r) / v(r)\right)<\infty$,

(iii) There are $\beta, r_{0}>0$ such that $v(r) e^{\beta r}$ is increasing on $\left[r_{0}, \infty[\right.$.

Proof. Let $r_{n}$ be a global maximum point of $r^{n} v(r)$.

(i) $\Rightarrow$ (ii): Theorem 2.1(b) implies $n / r_{n}=-v^{\prime}\left(r_{n}\right) / v\left(r_{n}\right) \leq \beta$ for some $\beta$ and large enough $n \in \mathbb{Z}_{+}$. Now fix some $r$, say $r_{n} \leq r \leq r_{n+1}$ for some $n \in \mathbb{Z}_{+}$. According to our assumptions on $v$ there is $m \in[n, n+1]$ such that $r$ is a maximum point for the function $s^{m} v(s)$. We have

$$
-n \frac{v\left(r_{n}\right)}{v^{\prime}\left(r_{n}\right)}=r_{n} \leq r=-m \frac{v(r)}{v^{\prime}(r)} \text {. }
$$

This implies

$$
-\frac{v^{\prime}(r)}{v(r)} \leq \frac{m}{n} \beta \leq \frac{n+1}{n} \beta
$$

and hence (ii).

(ii) $\Leftrightarrow\left(\right.$ iii): Put $f(r)=\log \left(v(r) e^{\beta r}\right)$. Then $f^{\prime}(r)=v^{\prime}(r) / v(r)+\beta$. Hence $v(r) e^{\beta r}$ is increasing if and only if (ii) holds. This proves the claim.

(ii) $\Rightarrow(\mathrm{i})$ : We proceed as in the proof of 2.1 to show that $D: H v(\mathbb{C}) \rightarrow$ $H v(\mathbb{C})$ is bounded. Fix $n$ and consider the operator $T_{n}$ of $(8)$. We have

$$
\left(T_{n} D h\right)(z)=\frac{k_{n+1}}{t_{n}}\left(U_{-1}\left(\mathrm{id}-\sigma_{k_{n+1}}\right) U_{1} T_{n} U_{-1} h\right)(z)
$$

if $|z|=t_{n}$. We claim that $k_{n+1} / t_{n}$ is uniformly bounded. First, (ii) implies

$$
\frac{n}{r_{n}}=-\frac{v^{\prime}\left(r_{n}\right)}{v\left(r_{n}\right)} \leq \beta
$$


for all $n>0$ and some $\beta>0$. Hence $n / \beta \leq r_{n}$. We may take $\beta$ so large that it satisfies the assertion of (iii) as well. Now fix $n$ and assume $k_{n+1} / \beta \geq t_{n}$. Using (9) with $h(z)=z^{k_{n+1}}$ we see that

$$
\begin{aligned}
c_{2} & \geq\left(\frac{r_{k_{n+1}}}{t_{n}}\right)^{k_{n+1}} \frac{v\left(r_{k_{n+1}}\right)}{v\left(t_{n}\right)} \geq\left(\frac{k_{n+1}}{\beta t_{n}}\right)^{k_{n+1}} \frac{v\left(k_{n+1} / \beta\right)}{v\left(t_{n}\right)} \\
& \geq\left(\frac{k_{n+1}}{\beta t_{n}}\right)^{k_{n+1}} \exp \left(\beta\left(t_{n}-\frac{k_{n+1}}{\beta}\right)\right) .
\end{aligned}
$$

Here the second inequality follows by comparing the function $r^{k_{n+1}} v(r)$ at $r=k_{n+1} / \beta$ and at the maximum point $r=r_{k_{n+1}}$. Using (iii), since $k_{n+1} / \beta \geq$ $t_{n}$ we obtain the last inequality.

Hence

$$
\frac{k_{n+1}}{t_{n}} \leq \beta c_{2}^{1 / k_{n+1}} \exp (1) .
$$

If $k_{n+1} / \beta \leq t_{n}$ then $k_{n+1} / t_{n} \leq \beta$. So (11) together with (10) imply

$$
\begin{aligned}
M_{\infty}\left(T_{n} D h, t_{n}\right) v\left(t_{n}\right) \leq & \widetilde{c} \frac{k_{n+1}}{t_{n}} M_{\infty}\left(U_{1} T_{n} U_{-1} h, t_{n}\right) v\left(t_{n}\right) \\
\leq & \widetilde{c} \frac{k_{n+1}}{t_{n}}\left(M_{\infty}\left(T_{n} h, t_{n}\right)\right. \\
& +M_{\infty}\left(T_{n}-U_{1} T_{n} U_{-1} h, t_{n}\right) v\left(t_{n}\right) \\
\leq & c \beta\left(M_{\infty}\left(T_{n} h, t_{n}\right)+M_{\infty}\left(h, t_{n}\right)\right) v\left(t_{n}\right)
\end{aligned}
$$

where $\widetilde{c}$ and $c$ are universal constants. Here we used again the fact that $U_{k}$ and $\sigma_{k_{n+1}}$ are uniformly bounded with respect to $M_{\infty}\left(\cdot, t_{n}\right)$ and the operator norms do not depend on $n$. Finally, (8) shows that $D$ is bounded.

4.2. Theorem. Let $v:[0, \infty[\rightarrow] 0, \infty[$ be a weight. Then the following are equivalent:

(i) $h \in H v(\mathbb{C})$ if and only if $h^{\prime} \in H v(\mathbb{C})$.

(ii) $D: H v(\mathbb{C}) \rightarrow H v(\mathbb{C})$ is bounded and surjective.

(iii) There are $c_{1}, c_{2}>0$ such that, for all $h \in H v(\mathbb{C})$,

$$
c_{1}\left(\left\|h^{\prime}\right\|_{v}+|h(0)|\right) \leq\|h\|_{v} \leq c_{2}\left(\left\|h^{\prime}\right\|_{v}+|h(0)|\right) .
$$

(iv) $0<\liminf _{r \rightarrow \infty}\left(-v^{\prime}(r) / v(r)\right) \leq \lim \sup _{r \rightarrow \infty}\left(-v^{\prime}(r) / v(r)\right)<\infty$.

(v) There are $\alpha, \beta, r_{0}>0$ such that $v(r) e^{\alpha r}$ is decreasing and $v(r) e^{\beta r}$ is increasing on $\left[r_{0}, \infty[\right.$.

Proof. The first three items are equivalent by the closed graph theorem and the open mapping theorem.

Let $r_{n}$ be a global maximum point of $r^{n} v(r)$.

(iv) $\Leftrightarrow(\mathrm{v})$ : Put $f(r)=\log \left(v(r) e^{\alpha r}\right)$ and $g(r)=\log \left(v(r) e^{\beta r}\right)$. Then we have $f^{\prime}(r)=v^{\prime}(r) / v(r)+\alpha$ and $g^{\prime}(r)=v^{\prime}(r) / v(r)+\beta$. Hence $v(r) e^{\alpha r}$ is 
decreasing if and only if $-v^{\prime}(r) / v(r) \geq \alpha$, and $v(r) e^{\beta r}$ is increasing if and only if $-v^{\prime}(r) / v(r) \leq \beta$. This proves the claim.

(ii) $\Rightarrow$ (iv): That $\limsup _{r \rightarrow \infty}\left(-v^{\prime}(r) / v(r)\right)<\infty$ follows from Theorem 4.1. Proposition 2.2(b) yields $0<\liminf _{n \in \mathbb{Z}_{+}, n \rightarrow \infty}\left(-v^{\prime}\left(r_{n}\right) / v\left(r_{n}\right)\right)$. Fix $r>0$ and $n \in \mathbb{Z}_{+}$such that $r_{n-1} \leq r \leq r_{n}$. Then $r$ is a global maximum point of $t^{m} v(t)$ for some $m \in[n-1, n]$. We have

$$
-n \frac{v\left(r_{n}\right)}{v^{\prime}\left(r_{n}\right)}=r_{n} \geq r=-m \frac{v(r)}{v^{\prime}(r)}
$$

which implies

$$
-\frac{v^{\prime}(r)}{v(r)} \geq-\frac{m}{n} \frac{v^{\prime}\left(r_{n}\right)}{v\left(r_{n}\right)} \geq-\frac{n-1}{n} \frac{v^{\prime}\left(r_{n}\right)}{v\left(r_{n}\right)} .
$$

This proves (iv).

(iv) $\Rightarrow$ (ii): According to Theorem 4.1, $D$ is bounded. Finally, we show that the integration operatotion $I: H v(\mathbb{C}) \rightarrow H v(\mathbb{C})$ is bounded. Fix $n$ and consider the operator $T_{n}$ of (8). By Lemma 3.1(b) of [16], we have

$$
M_{\infty}\left(T_{n} U_{1} h, s\right) \leq 2\left(\frac{s}{t_{n}}\right)^{k_{n-2}-1} M_{\infty}\left(T_{n} U_{1} h, t_{n}\right) \quad \text { if } t_{n} \geq s .
$$

Hence

$$
\begin{aligned}
M_{\infty}\left(T_{n} I h, t_{n}\right) v\left(t_{n}\right) \leq & \int_{0}^{t_{n}} M_{\infty}\left(T_{n} U_{1} h, s\right) d s v\left(t_{n}\right) \\
\leq & 2 M_{\infty}\left(T_{n} U_{1} h, t_{n}\right) \int_{0}^{t_{n}}\left(\frac{s}{t_{n}}\right)^{k_{n-2}-1} d s v\left(t_{n}\right) \\
\leq & 2 M_{\infty}\left(U_{-1} T_{n} U_{1} h, t_{n}\right) \frac{t_{n}}{k_{n-1}} v\left(t_{n}\right) \\
\leq & c\left(M_{\infty}\left(T_{n} h, t_{n}\right)\right. \\
& \left.+M_{\infty}\left(T_{n}-U_{-1} T_{n} U_{1} h, t_{n}\right)\right) \frac{t_{n}}{k_{n-2}} v\left(t_{n}\right) \\
\leq & c\left(M_{\infty}\left(T_{n} h, t_{n}\right)+M_{\infty}\left(h, t_{n}\right)\right) \frac{t_{n}}{k_{n-2}} v\left(t_{n}\right)
\end{aligned}
$$

where $c$ is a universal constant. (We have used (10).)

We claim that $t_{n} / k_{n-2}$ is uniformly bounded. Fix $n$ and let $\alpha$ be the constant of (v). Using (9) with $h(z)=z^{k}$ where $k=k_{n-2}+1$ we see that, in view of (iv),

$$
\begin{aligned}
c_{2} & \geq\left(\frac{r_{k}}{t_{n}}\right)^{k} \frac{v\left(r_{k}\right)}{v\left(t_{n}\right)} \geq\left(\frac{t_{n}}{2 t_{n}}\right)^{k} \frac{v\left(t_{n} / 2\right)}{v\left(t_{n}\right)} \\
& \geq\left(\frac{1}{2}\right)^{k} \exp \left(\alpha\left(t_{n}-t_{n} / 2\right)\right)=\exp \left(-k \log 2+\alpha t_{n} / 2\right)
\end{aligned}
$$


For the last inequality we have used $v\left(t_{n} / 2\right) e^{\alpha t_{n} / 2} \geq v\left(t_{n}\right) e^{\alpha t_{n}}$, which holds according to (v). So, for large $n$ we have $t_{n} \leq 3 \alpha^{-1} k \log 2$ and hence $t_{n} / k_{n-2}$ is uniformly bounded. Now (12) together with (8) shows that $I$ is bounded.

Of course the standard example for Theorem 4.1 is $v(r)=e^{-r}$. Moreover, for $v(r)=\exp \left(-\log ^{2} r\right)$ the differentiation operator $D: H v(\mathbb{C}) \rightarrow H v(\mathbb{C})$ is bounded (in view of Theorem 2.1) but not surjective. For $v(r)=\exp \left(-e^{r}\right)$ the differentiation operator is unbounded since

$$
\lim _{n \rightarrow \infty}-v^{\prime}\left(s_{n}\right) / v\left(s_{n}\right)=\lim _{n \rightarrow \infty} e^{s_{n}}=\infty
$$

for the global maximum points $s_{n}$ of $r^{n} v(r)$ (Lemma 1.2).

\section{References}

[1] B. Berndtsson and J. Ortega Cerdà, On interpolation and sampling in Hilbert spaces of analytic functions, J. Reine Angew. Math. 464 (1995), 109-128.

[2] O. Blasco and A. Galbis, On Taylor coefficients of entire functions integrable against exponential weights, Math. Nachr. 223 (2001), 5-21.

[3] K. Bogalska, Multiplication operators on weighted Banach spaces of analytic functions with exponential weights, Bull. Polish Acad. Sci. Math. 49 (2001), 409-416.

[4] J. Bonet, Weighted spaces of holomorphic functions and operators between them, Colecc. Abierta 64, Univ. Sevilla Secr. Publ., Seville, 2003, 117-138.

[5] J. Bonet, P. Domański and M. Lindström, Essential norm and weak compactness of composition operators on weighted Banach spaces of analytic functions, Canad. Math. Bull. 42 (199), 139-148.

[6] - - - - , Pointwise multiplication operators on weighted Banach spaces of analytic functions, Studia Math. 137 (1999), 177-194.

[7] J. Bonet, P. Domański, M. Lindström and J. Taskinen, Composition operators between weighted spaces of analytic functions, J. Austral. Math. Soc. 64 (1998), 101118.

[8] J. Bonet and E. Wolf, A note on weighted Banach spaces of holomorphic functions, Arch. Math. (Basel) 81 (2003), 650-654.

[9] K. Cichoń and K. Seip, Weighted holomorphic spaces with trivial closed range multiplication operators, Proc. Amer. Math. Soc. 131 (2003), 201-207.

[10] P. Domański and M. Lindström, Sets of interpolation and sampling for weighted Banach spaces of holomorphic functions, Ann. Polon. Math. 89 (2002), 233-264.

[11] A. Galbis, Weighted Banach spaces of entire functions, Arch. Math. (Basel) 62 (1994), 58-64.

[12] W. Kaballo, Lifting-Probleme für $H^{\infty}$-Funktionen, ibid. 34 (1980), 540-549.

[13] W. Lusky, Growth conditions for harmonic and holomorphic functions, in: Functional Analysis (Trier, 1994), S. Dierolf et al. (eds.), de Gruyter, 1996, 281-291.

[14] - On weighted spaces of harmonic and holomorphic functions, J. London Math. Soc. 59 (1995), 309-320.

[15] -, On the Fourier series of unbounded harmonic functions, ibid. 61 (2000), 568-580.

[16] - On the isomorphism classes of weighted spaces of harmonic and holomorphic functions, Studia Math. 75 (2006), 19-45.

[17] K. Seip, Density theorems for sampling and interpolation in the Bargmann-Fock space, Bull. Amer. Math. Soc. 26 (1992), 322-328. 
[18] K. Seip, Beurling type density theorems in the unit disc, Invent. Math. 113 (1993), $21-39$.

[19] A. L. Shields and D. L. Williams, Bounded projections, duality and multipliers in spaces of analytic functions, Trans. Amer. Math. Soc. 162 (1971), 287-302.

[20] - - - Bounded projections, duality and multipliers in spaces of harmonic functions, J. Reine Angew. Math. 299/300 (1978), 256-279.

[21] - - - Bounded projections and the growth of harmonic conjugates in the disc, Michigan Math. J. 29 (1982), 3-25.

[22] J. Taskinen, Compact composition operators on general weighted spaces, Houston J. Math. 27 (2001), 203-218.

Faculty for Informatics and Applied Mathematics

Institute for Mathematics

University of Yerevan

University of Paderborn

Alek Manukian 1

Yerevan 25, Armenia

E-mail: anahit@ysu.am

Warburger Str. 100

D-33098 Paderborn, Germany E-mail: lusky@uni-paderborn.de

Received December 8, 2006

Revised version November 16, 2007

$(6065)$ 